\section{Using a Focused Ion Beam (FIB) System to Extract TEM-Ready Samples from Complex Metallic and Ceramic Structures}

Lucille A. Giannuzzi, University of Central Florida

Richard Young and Pete Carleson, FEI Company

Fred Stevie, Cirent Semiconductor (a partnership with Lucent Technologies)

\section{Abstract}

Driven by the analytical needs of microelectronics, magnetic media and micro-fabrication industries, focused ion beam (FIB) systems are now capable of milling and manipulating samples for the analysis of microstructure features having dimensions of $180 \mathrm{~nm}$ or less. A technique for locating and extracting site specific specimens for examination by transmission electron microscopy (TEM) has been developed. An identified feature can be located and precisely milled with an FIB system from two sides to prepare an ultrathin sample, and then extracted from the region with a glass rod micromanipulator onto a grid for TEM analysis. This specimen preparation method has been applied to semiconductor failure analysis and to the study of metallic and ceramic microstructures with irregular topographies and complex multi-layered components.

\section{Introduction}

Transmission electron microscopes are commonly used to study biological, metallurgical, ceramic, and crystalline structures. By viewing a specimen with a TEM $M_{1}$ one can determine elemental composition within a spatial resolution of $<1 \mathrm{~nm}$, analyze crystal lattice structures, and study complex microstructures. Specimens prepared for TEM analysis must be small enough (typically $3 \mathrm{~mm}$ in diameter or less) to fit into the microscope specimen holder, and thin enough $(<\sim 100 \mathrm{~nm})$ to be electron transparent.

Preparing a suitable TEM specimen is often the limiting factor in performing TEM analysis. Many specimen preparation techniques may require "artistic" skills for success. In some instances, recipes that are successful in one laboratory may not work in another. Using a focused ion beam (FIB) instrument, one can produce TEM specimens routinely, quickly, and precisely from a wide range of materials ${ }^{1}$.

\section{The Lift-Out Technique}

The "lift-out" technique was developed initially for microelectronics device applications. A TEM specimen can be extracted while the high-value semiconductor wafer remains intact. The method was first described by a group at Philips ${ }^{2}$ and further developed at Cirent Semiconductor and the University of Central Florida ${ }^{3}$. The specimen preparation technique described here was developed on integrated circuits and other metallic and ceramic

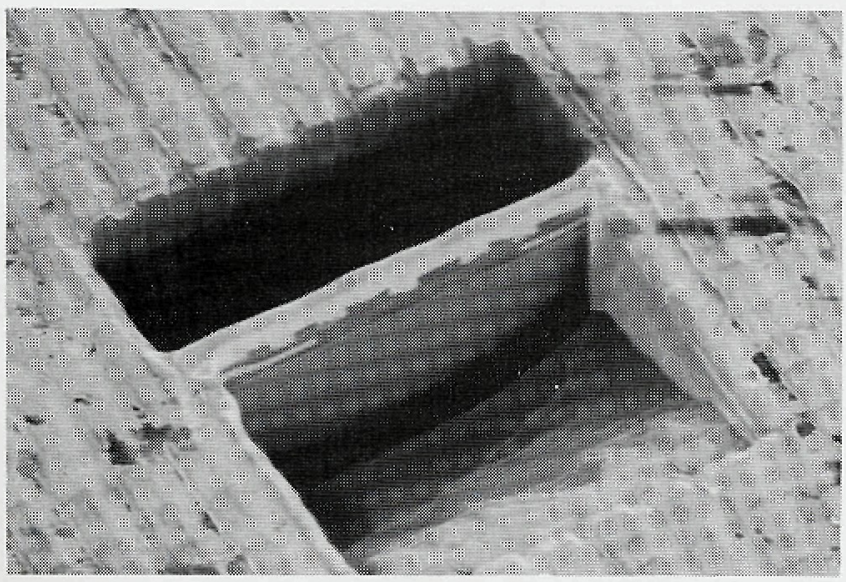

Figure 1: FIB image of specimen after the bulk material removal steps are completed. microstructures utilizing a FEI FIB ion milling system. TEM analysis was performed on a Philips EM430 operating at $300 \mathrm{kV}$. Only the outline of the experimental technique is presented; a more detailed description has been published previously ${ }^{3}$.

A bulk sample, such as a $200 \mathrm{~mm}$ diameter silicon wafer, is placed directly into the vacuum chamber of the FIB for TEM specimen preparation. Using control and navigation software provided with the $F \mid B$ tool, the site of interest, such as a particular circuit, interface layer, metal bonding site, or potential defect area, is located on the sample surface. The local area to be cross-sectioned is coated with a protective layer of tungsten or platinum using ion beam induced deposition. Bulk material is removed by milling trenches parallel to each side of the protective film until a film thickness of approximately $300 \mathrm{~nm}$ is reached. Figure 1 is a view of the specimen after the platinum film and bulk material removal steps have been completed. The FIB system can also use reactive gas-assisted etching that accelerates the milling rate.

A high ion beam current is used for the initial bulk milling, while a lower beam current is used for the final thinning of the specimen to an electron transparent membrane. The diameter of the gallium ion sputtering beam can be below $10 \mathrm{~nm}$ and can be positioned with accuracy of less than $50 \mathrm{~nm}$. The operator can visually monitor the thinning process and make adjustments as necessary. Highresolution secondary-electron images can be obtained during the process if desired. The wafer is tilted to 60 degrees, exposing the base and sides of the film. Subsequent FIB cuts partially separate the specimen section from the bulk material. Figure 2 shows the $300 \mathrm{~nm}$ thick sample severed at the bottom and sides. The sample is tilted back to 0 degrees and the film is further thinned to the desired thickness, usually $\sim 100 \mathrm{~nm}$ for conventional TEM analysis. A final FIB release cut is made and the membrane is freed from the bulk sample, ready for extraction (Figure 3).

\section{Sample Extraction}

Once the membrane has been cut away, the entire bulk sample is then removed from the FIB and placed under an optical microscope. A glass rod or tube drawn to a small tip and attached to a micromanipulator arm is used to transfer the TEM thin membrane from the bulk sample to a TEM grid. It is believed that electrostatic forces attract the membrane to the glass pipette for safe transport to a carbon or Formvar-coated copper TEM analysis grid. Figure 4 shows an optical microscope and micromanipulator for extracting the specimen from the intact wafer. Figure 5 is a scanning electron micrograph of a lift-out specimen positioned on a TEM grid

\section{Discussion}

Figure 6 is a bright field TEM image of an integrated circuit cross section Continued on page 14

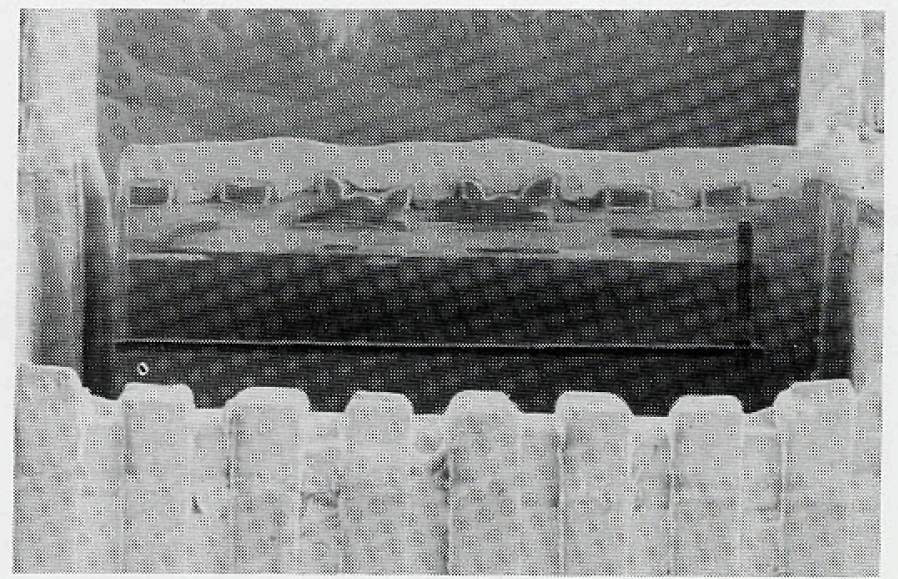

Figure 2: FIB image of specimen thinned to $300 \mathrm{~nm}$ and severed at the bottom and sides. 


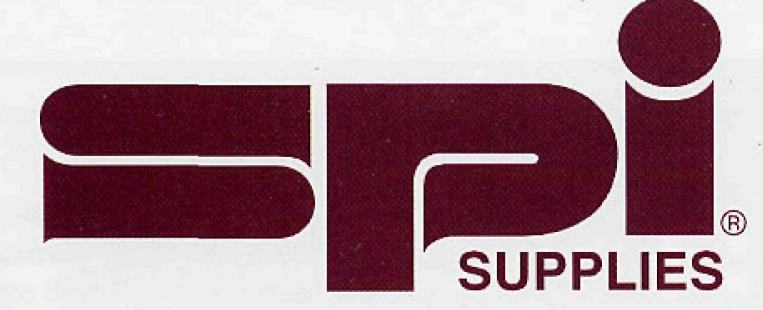

File Edit Diew Go Bookmarks 0ptions Directory Help

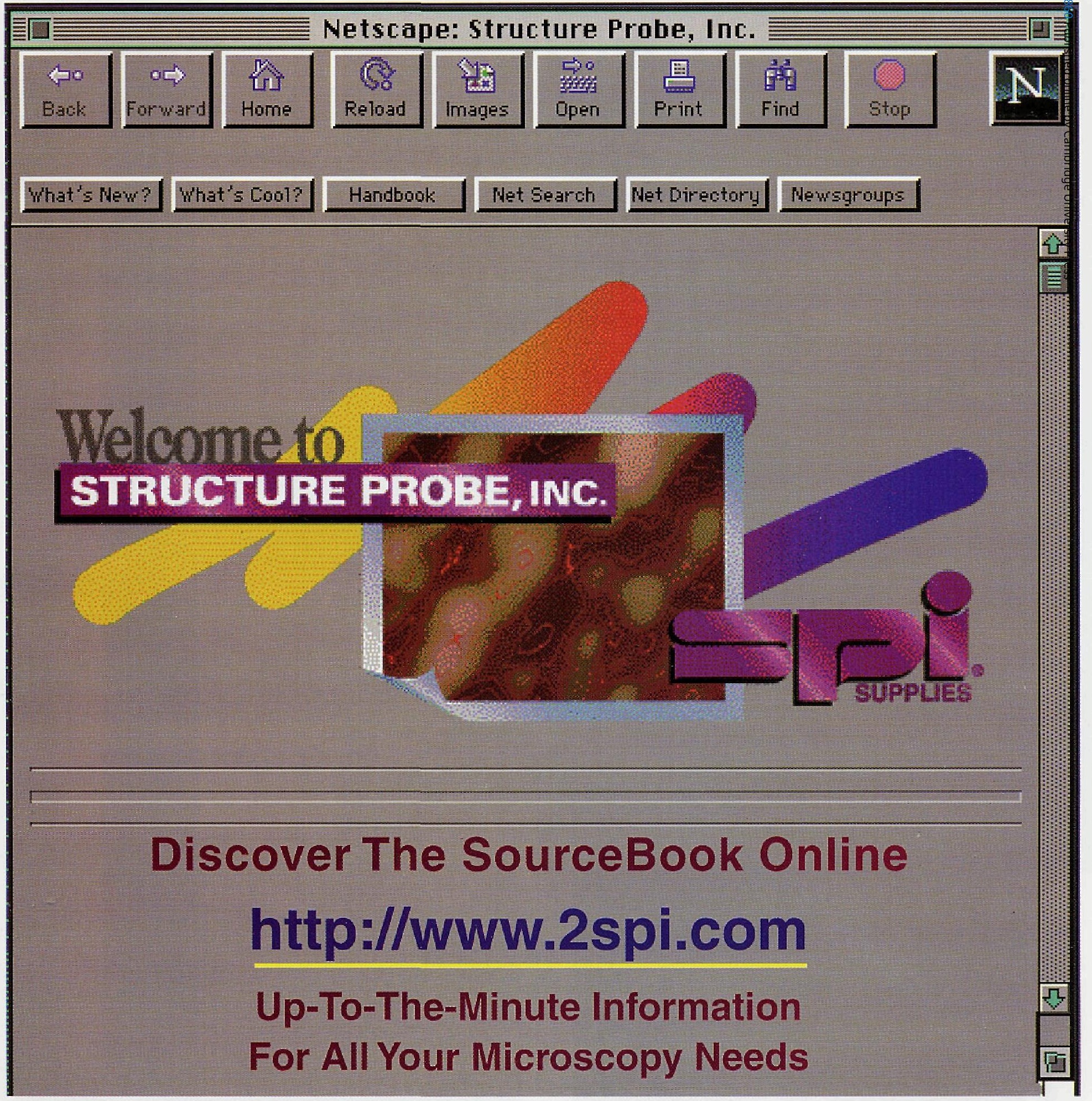

SPI Supplies Division of STRUCTURE PROBE, Inc.

P.O. Box 656 - West Chester, PA 19381-0656 USA 


\section{Using a Focused Ion Beam (FIB) System...}

Continued from page 12

that was prepared by the FIB lift-out technique

A major benefit in performing an entire specimen preparation in a FIB tool is the time savings. Prior methods, which required movement of the bulk sample from machine to machine for different stages of the process, could take several hours and much longer for difficult specimens. The development of TEM specimen preparation techniques for new materials may consume days, weeks, or even years of research. In contrast, the majority of specimen preparations conducted to date with a FIB tool with the technique outlined here have taken at most 5 hours. Recent developments in FIB instrumentation allow much of the process to be automated and run unattended4. The vacuum environment of the FIB tool also minimizes the potential contamination of samples; this is important for the examination of sub-micrometer features.

\section{Applications}

Advances in focused ion beam technologies and their incorporation in commercially available systems is expanding the realm of microstructures that can be manipulated and analyzed. The FIB-based specimen preparation and extraction technique provides scientists and researchers with a new method for extracting TEM specimens from materials and from layered metal or ceramic structures with complex atomic interfaces or irregular topographies. This technique can be applied to preparing TEM specimens for quality control of manufacturing processes, failure analysis, or research and devel-

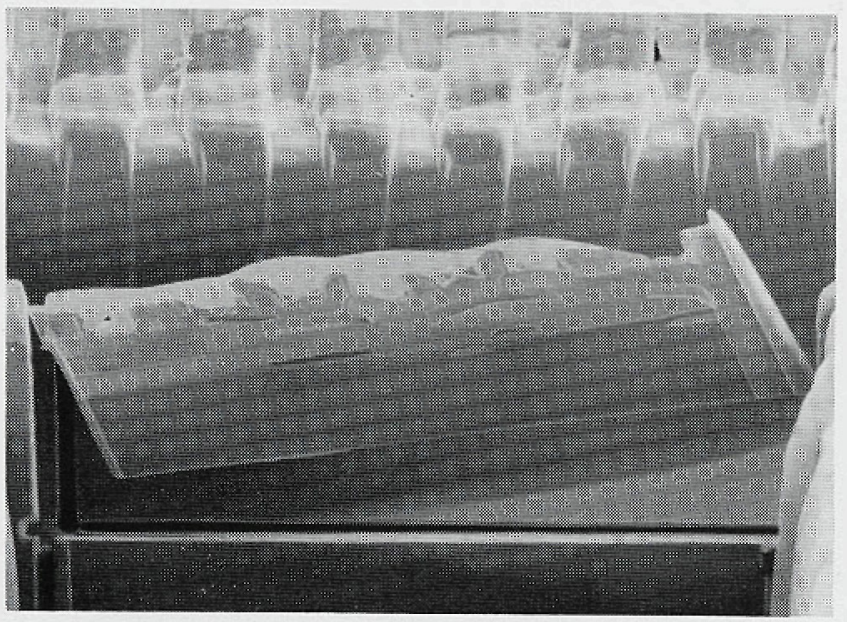

Figure 3: FIB image of a specimen ready for extraction after release from the whole wafer.

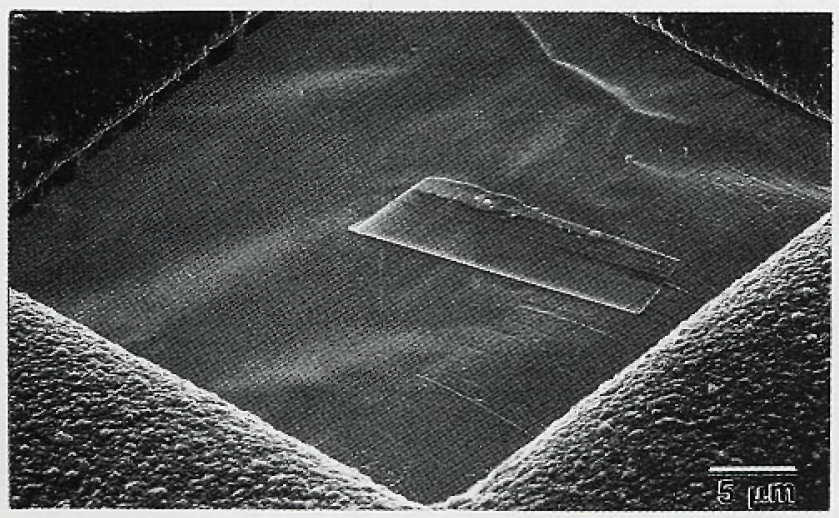

Figure 5: SEM image of a lift-out specimen positioned on a TEM grid. opment of new materials for a wide range of industries.

\section{Summary}

Modern FIB tools are ideal for TEM specimen preparation for microelectronics and other difficult materials. The increased accuracy of on-wafer navigation, beam placement for FIB cuts, and finer beam resolution enables the precision identification and extraction of higher quality specimens having dimensions of $100 \mathrm{~nm}$ or less with consistent uniformity. TEM specimens may be produced without destructive sample preparation of the bulk material. These advances in FIB tool capabilities also expand the use of the TEM for the exploration of new materials and microstructures.

\section{Acknowledgments}

We wish to acknowledge contributions to this work of $R$. Irwin and Steve Brown at Cirent Semiconductor and J. L. Drown and B. I. Prenitzer of the University of Central Florida.

\section{References}

[1] L.A. Giannuzzi, J.L. Drown, S.R. Brown, R.B. Irwin, and F.A. Stevie, Microscopy Research and Technique, 41:285-290 (1998).

[2] M.H.F. Overwijk, F.C. van den Heuvel, and C.W.T. Bulle-Lieuwma, J. Vac. Sci. Technol. B11, 2021 (1993).

[3] L.A. Giannuzzi, J.L. Drown, S.R. Brown, R.B. Irwin, F.A. Stevie, Mat. Res. Symp.Proc. 480, 19 (1997), Materials Research Society.

[4] R.J. Young, P.D. Carleson, X. Da, T. Hunt, J.F. Walker, Proceedings International Symposium for Testing and Failure Analysis, 329-336 (1998).

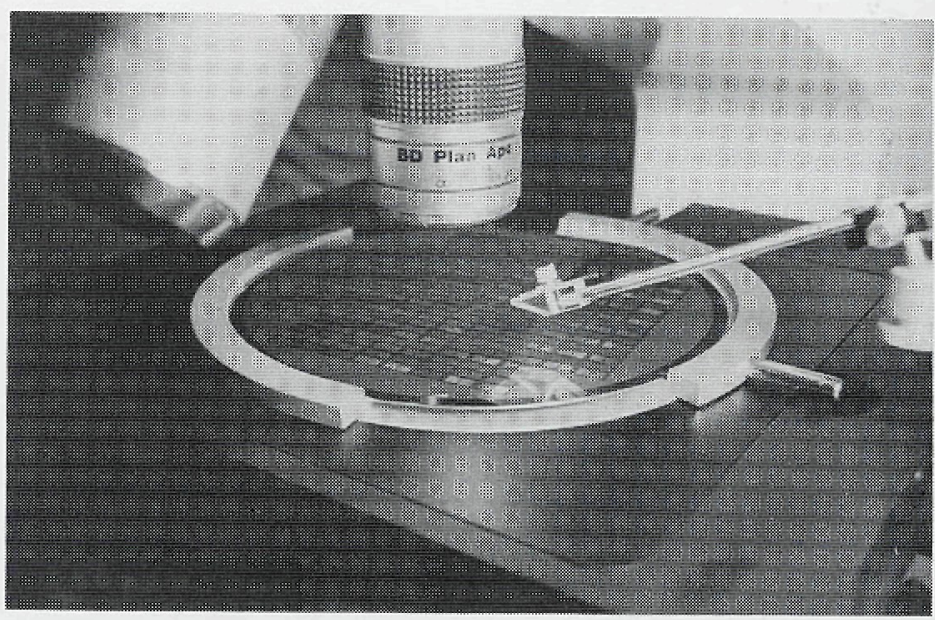

Figure 4: Extraction of sample from intact wafer by micromanipulator.

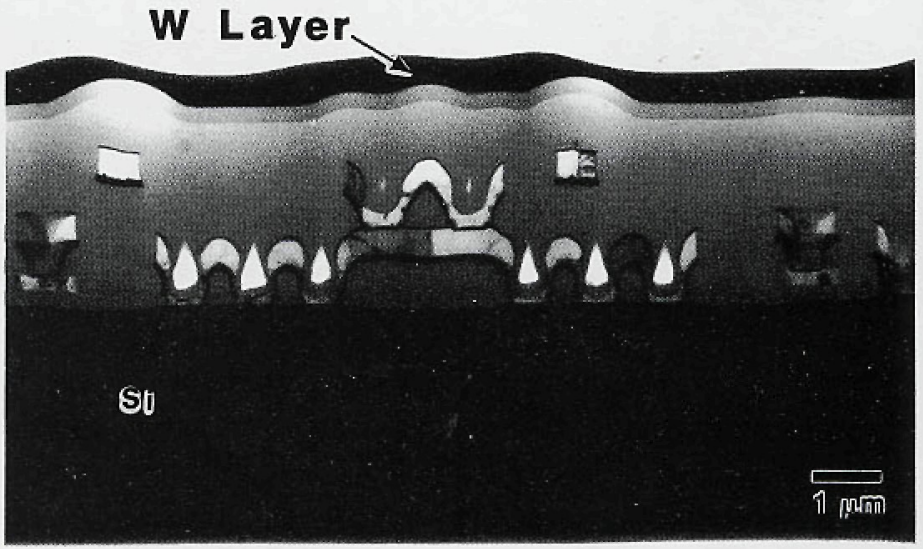

Figure 6: TEM image of an integrated circuit prepared by the FIB lift-out technique. 


\section{FTHIR}
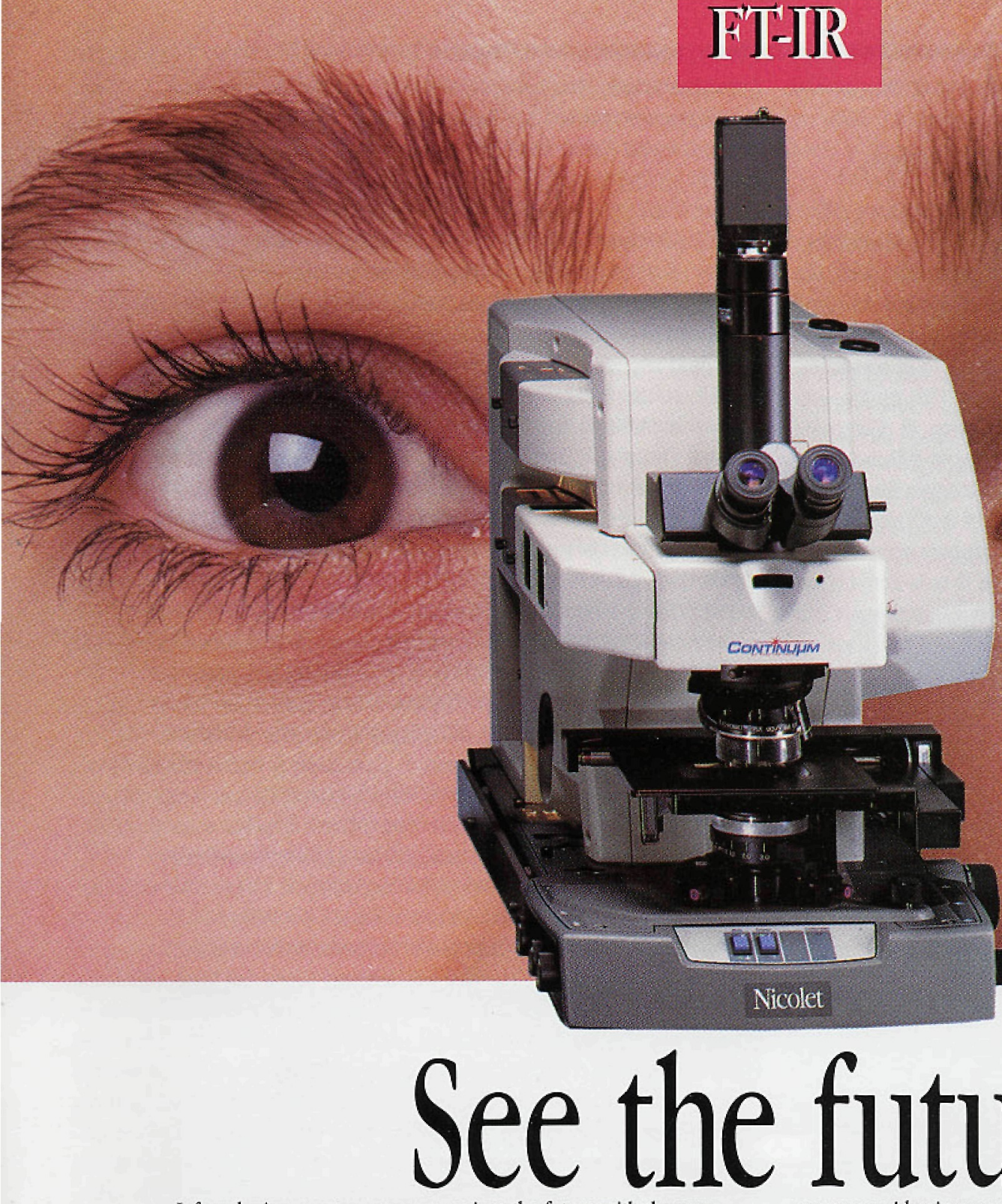

Infrared microspectroscopy moves into the future with the new Continuum ${ }^{\text {tw }}$ microscope.

With exclusive infinity corrected optics, you'll get visual images superior to anything on the market today. And thanks to patented dual remote aperturing, the spectrum you collect represents the image you see.

Use the unique OMNIC ${ }^{\text {At }}$ tlus ${ }^{\text {Th }}$ software. You will see how easy and clear data analysis can be with InterLinking, which links the

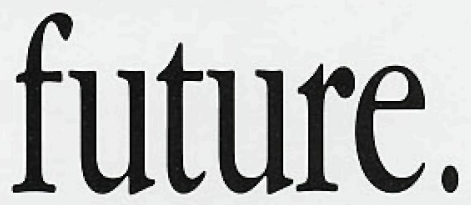

spectrum, video image, composition maps and 3D images. In addition, the easy-to-use Continuum is fully automated. So everything from collection to mapping can be completed with consistency and precision.

The Continuum is more than new technology. Developed by Spectra-Tech exclusively for Nicolet, it is the world's first fully upgradeable IR microscope. It evolves as your needs change, allowing you to get answers to your most difficult questions. To learn more about Continum, contact Nicolet. Let us show you the future.

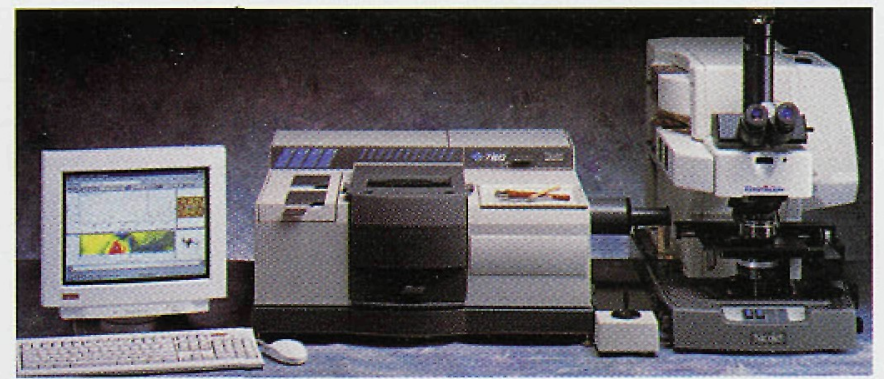

Magna-IR ${ }^{\circ} 760$ E.S.P. Spectrometer with Continuum Microscope
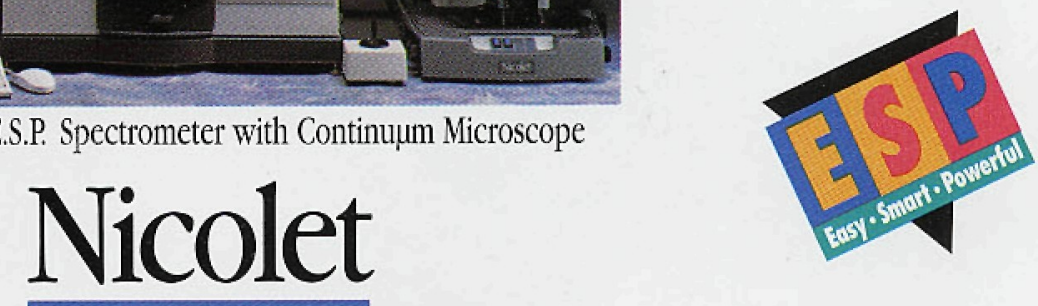

INSTRUMENTS OF DISCOVERY

A sulssidiary of Thermo Optek Corporation, a Thermo Instrument Systens, Inc. company 